\title{
Optimal pattern of technology adoptions under embodiment: A multi-stage optimal control approach
}

\author{
Cagri Saglam*, ${ }^{*}$ \\ Department of Economics, Bilkent University, Ankara, Turkey
}

\begin{abstract}
SUMMARY
By deriving the necessary conditions for a multi-stage optimal control problem where the endogenous switching instants appear as an argument of the state equation, we analyze the optimal pattern of technology adoptions under embodiment with a finite planning horizon. We show that the optimal pattern of technology adoptions depends crucially on how the growth rate advantage compares to the adjustment and the obsolescence costs inherent to embodiment. We obtain non-stationary lifetimes for the adopted technologies due to finite planning horizon. We analyze numerically the effects of planning horizon, speed of adjustment to the new technology, growth rate of technology and the impatience rate on the optimal pattern. Copyright (C) 2010 John Wiley \& Sons, Ltd.
\end{abstract}

Received 22 August 2008; Revised 6 June 2010; Accepted 9 July 2010

KEY WORDS: multi-stage optimal control; technology adoption; embodiment

\section{INTRODUCTION}

This paper characterizes a theoretical framework to analyze the optimal timing and pattern of technology adoptions under embodiment over the course of a finite planning horizon. Since the system underlying such an individual problem of technology adoption and investment involves abrupt discrete switches, we propose a multi-stage optimal control approach where the switching instants are endogenously determined.

This paper contributes to the theory through the derivation of necessary conditions of optimality for multi-stage control problems in which the endogenous switching times appear as an argument of the state equations. Early contributions on the control of such systems have been mainly limited with two-stage optimal control techniques that recall Pontryagin maximum principle from a dynamic programming perspective (see [1,2]). Makris [3] extended the analysis to allow for multiple regime switches but has not considered the fact that the switch-points may appear as an argument of the state equations. The illustrations of two-stage optimal control on technology adoption problems can be found in Boucekkine et al. [4]. In this respect, our theory both harnesses and extends the analysis of Boucekkine et al. [4] by analyzing multiple technology switches.

We concentrate on a model of life-cycle technology adoption in which the agent faces a technology frontier that evolves exogenously at a constant rate. Newer technology implies a larger investment-specific productivity and, as such, adoption of it represents an acceleration in embodied technical change. At any instant of time, the agent may either upgrade to the frontier level of capital accumulation technology or continue to use the current one. However, adoption of a new technology is costly as more embodiment implies a decrease in the relative price of capital, which

\footnotetext{
${ }^{*}$ Correspondence to: Cagri Saglam, Department of Economics, Bilkent University, Ankara, Turkey.

†E-mail: csaglam@bilkent.edu.tr
} 
induces a re-assignment of the resources towards investment, resulting in a drop in the consumption level. The welfare cost of this drop in the consumption level is referred to as obsolescence costs and they are shown to be non-negligible (see [4-6]). In addition to these obsolescence costs inherent to embodiment, switching to a newer technology induces an accelerated erosion effect on physical capital (see $[7,8]$ ) and a slow adjustment process for reaching the best productivity level of the new technology. This posits a trade-off between the improvement in the efficiency of investment, referred to as growth rate advantage and the adjustment cost of technology adoption together with obsolescence inherent to embodiment. In such an environment, the agent seeks to maximize the standard discounted sum of utility from consumption over a finite time interval by choosing the number, and the timing of technology upgrades, and the path for consumption.

Considering optimal pattern of technology adoption and the trade-offs therein, our theory touches upon the works by Parente [9,10], Mateos-Planas [11] and Boucekkine et al. [12]. The cost of adoption in all these studies is mainly the loss of expertise, namely the specific human capital accumulated on the currently used technique. Parente analyzes the optimal adoption problem with an infinite planning horizon and conjectures that the optimal adoption plans are typically characterized by evenly spaced pattern of technology adoptions. In such a framework, there is no possibility to stick to a given technology so that there will be no room to handle technological sclerosis. Mateos-Planas [11] incorporates a finite planning horizon and predicts a non-stationarity in the sense that an optimal plan may incorporate both types of adopted technologies that are to be learned and that are to be skipped without learning. The model considers the adoption problem of an agent operating a single firm that produces output using one machine and leaves no room to deal with the effects of technology adoption on the capital accumulation process. It adopts a step-wise discrete learning process and concludes that the adoptions may become more frequent as the agent grows older.

Our framework allows us to bring out a number of contributions to the optimal adoption literature as well. Following Boucekkine et al. [4], the optimal pattern of technology adoptions is determined by taking into account the non-negligible obsolescence cost inherent to embodiment. We characterize the optimal number of technology upgrades and the optimal timing of them within a given finite planning horizon. In contrast with models by Parente and its extensions, we obtain a non-stationarity in the durations of the adopted technologies to be in use due to the finite planning horizon. We note that the lifetimes of the technologies in use follow a monotonically increasing path as it is optimal to devote more time for using later technologies that are endowed with a higher level of investment efficiency and will eventually provide a higher level of consumption. In contrast with Mateos-Planas [11], the admissible plans that incorporate more frequent technology switches through the end of the planning horizon are not optimal. We illustrate how the optimal pattern of technology adoptions evolves with respect to the planning horizon, the speed of adjustment to new technology, the rate of growth of technology and the discount rate through numerical analysis.

This paper is organized as follows. Section 2 describes the model and optimal technology adoption problem. Section 3 presents the multi-stage optimal control problem as a resolution process. Section 4 illustrates the optimal pattern of technology adoption and how it responds to the changes in the parameters of the model with a numerical analysis. Finally, Section 4 concludes.

\section{THE MODEL}

We consider an individual problem of technology adoption and investment with a finite planning horizon. At an instant $t$, the agent produces the flow of output $A K(t)$, where $A>0$ denotes the marginal productivity of capital. Physical capital evolves as the result of investment according to

$$
\dot{K}(t)=q(t)(A K(t)-C(t))-\delta(t) K(t),
$$

where $C(t)$ denotes the flow of consumption. $q(t)$ denotes the efficiency of investment and, as such, it represents the embodied technical progress variable. This investment-specific productivity $q$ is determined by the technology in use, which corresponds to the frontier technology at the time 
that the technology was adopted by the agent. The frontier technology grows at a constant rate $\gamma$ and its value at time $t$ can be represented as $\mathrm{e}^{\gamma t}$ (see [10]).

A newer technology that has a higher $q$ associated with it implies a decrease in the relative price of capital and raises the user cost of capital by the so-called obsolescence costs $\$$. Apart from obsolescence cost inherent to embodiment, switching to a newer technology induces an accelerated erosion effect on physical capital (see [7]) and a slow adjustment process for reaching the best productivity level of the new technology. ${ }^{\S}$ Accordingly, the depreciation rate of the capital stock is composed of two parts, one of which incorporates the technology-specific adjustment cost of adoption. More precisely, we have:

$$
\delta(t)=\bar{\delta}+\eta q\left(t_{j}\right) \mathrm{e}^{-\theta\left(t-t_{j}\right)}, \quad \forall t \in\left[t_{j}, t_{j+1}\right),
$$

where $t_{j}$ denotes the switching instant of the $j$ th technology and $\eta \leqslant A$ and $\theta$ are positive parameters. In order to elaborate more on the cost of technology adoption, we will assume without any loss of generality that use-related physical deterioration rate, $\bar{\delta}$ is nil. Note that the accelerated loss of capital stock following a technology switch increases as the technological acceleration gets sharper. However, these adjustment costs are not permanent and asymptotically eliminated with a speed measured by the parameter $\theta$.

The agent seeks to maximize the standard discounted sum of utility from consumption over a finite time interval. The choice variables are the number, and the timing of technology upgrades, and the path for consumption. As the number of technology adoptions will also be chosen optimally, our analysis will constitute from two parts. In the first part, noting that the cost of switching limits the number of adoptions so that there can be zero, finite or countably infinite number of adoptions, we will present the necessary conditions for the optimal control of $n$ possible technology upgrades, where $n$ is an arbitrary-fixed number. Knowing that the arbitrary number of adoptions may not be consistent with the optimal choice, the second part of our analysis will be devoted to the determination of the optimal number of technology upgrades.

\subsection{Multi-stage optimal control problem}

We have the following multi-stage optimal control problem for a given sequence of switching, $t_{j-1} \leqslant t_{j}, j=1,2, \ldots, n$,

$$
\max _{\left\{t_{i}\right\}_{i=1}^{i=n}, c(t)} W\left(K(t), C(t), t_{1}, t_{2}, \ldots, t_{n}\right) \equiv \sum_{j=1}^{n+1} \int_{t_{j-1}}^{t_{j}} u(C(t)) \mathrm{e}^{-\rho t} \mathrm{~d} t
$$

subject to

$$
\begin{aligned}
\dot{K}(t)= & \begin{cases}q\left(t_{j-1}\right)\left[(A K(t)-C(t))-\eta \mathrm{e}^{-\theta\left(t-t_{j-1}\right)} K(t)\right], & \forall t \in\left[t_{j-1}, t_{j}\right), j=1, \ldots, n, \\
q\left(t_{n}\right)\left[(A K(t)-C(t))-\eta \mathrm{e}^{-\theta\left(t-t_{n}\right)} K(t)\right], & \forall t \in\left[t_{n}, t_{n+1}\right],\end{cases} \\
& C(t) \geqslant 0, \quad K(t) \geqslant 0, \quad \forall t \in\left[t_{0}, t_{n+1}\right], \\
& T<\infty \text { given and } K(t) \geqslant 0 \text { free, } \\
& \left\{t_{i}, K\left(t_{i}\right)\right\}, \quad i=1,2, \ldots, n \quad \text { free, } \\
& t_{0} \geqslant 0, \quad K\left(t_{0}\right)=K_{0} \quad \text { given, }
\end{aligned}
$$

\footnotetext{
\$This is a well-known property since Solow [13]. Indeed, if the planning horizon were infinite, the model would turn out to be the reduced form of Solow [13] vintage capital model $[4,12,14]$.

${ }^{\S}$ With an infinite planning horizon, Jovanovic and Nyarko [15], Parente [9,10] and Boucekkine et al. [12] also analyze the dilemma of whether to stick to an established technology or to adopt a new and better one. Mateos-Planas [11] considers a finite planning horizon. However, the cost of adoption in all of these studies is mainly the loss of expertise, namely the specific human capital accumulated on the currently used technique. See Greenwood and Jovanovix [16] for a survey on the costs of technology adoption.
} 
where $n$ denotes the given maximum number of technology upgrades. The discrete controls $t_{j}$ $(j=1,2, \ldots, n)$ determine the switching instants between alternative and consecutive regimes. The planning horizon is finite and we adopt the convention $t_{n+1}=T$. The state variable $K(t) \in \mathbb{R}_{+}$is one-dimensional, continuous and piecewise continuously differentiable function of $t \in\left[t_{0}, T\right]$ and its behavior is governed by the regime-specific differential Equation (2). The control variable $C(t)$ is a piecewise continuous function of $t \in\left[t_{0}, T\right]$ valued in $\mathbb{R}_{+}$. The intertemporal utility function $u: \mathbb{R}_{+} \rightarrow \mathbb{R}$ is strictly increasing, strictly concave and satisfies Inada condition: $u^{\prime}(0)=+\infty$.

We refer to $\left\{t_{1}, \ldots, t_{n}\right\}$ and $\{K(t), C(t)\}, t \in\left[t_{0}, T\right]$ as admissible whenever there exists a solution for the state equation that satisfies the initial and the boundary conditions and the value of the objective function is finite with $t_{j-1} \leqslant t_{j}, \forall j=1,2, \ldots, n+1$, A path $\left(K^{*}(t), C^{*}(t), t_{1}^{*}, \ldots, t_{n}^{*}\right)$ is optimal if it is admissible and we have

$$
W\left(K^{*}(t), C^{*}(t), t_{1}^{*}, \ldots, t_{n}^{*}\right) \geqslant W\left(K(t), C(t), t_{1}, t_{2}, \ldots, t_{n}\right)
$$

for any other admissible path $\left(K(t), C(t), t_{1}, t_{2}, \ldots, t_{n}\right)$.

The novel feature of the problem is that the endogenous switching instants appear explicitly as an argument of the law of motion of the state. It is important to note that early contributions by Tomiyama [1], Tomiyama and Rossana [2] and Makris [3] cannot be used to handle this optimization problem.

The following theorem presents the necessary conditions for the optimum.

\section{Theorem 1}

Suppose that $\left(K^{*}(t), C^{*}(t), t_{1}^{*}, \ldots, t_{n}^{*}\right)$ is the optimal path. Then, there exist continuous and piecewise continuously differentiable co-state functions, $\lambda_{j}(t), \forall t \in\left[t_{j-1}, t_{j}\right], j=1,2, \ldots, n+1$, such that (2) and the continuity conditions:

$$
\lambda_{i+1}\left(t_{i}^{*}\right)=\lambda_{i}\left(t_{i}^{*}\right), \quad i=1,2, \ldots, n,
$$

hold. Moreover, defining the Hamiltonian function for each regime as

$$
\begin{aligned}
H_{j}\left(t, K(t), C(t), \lambda_{j}(t), t_{j-1}\right)= & u(C(t)) \mathrm{e}^{-\rho t}+\lambda_{j}(t) q\left(t_{j-1}\right)\left[(A K(t)-C(t))-\eta \mathrm{e}^{-\theta\left(t-t_{j-1}\right)} K(t)\right], \\
& \forall t \in\left[t_{j-1}, t_{j}\right), j=1, \ldots, n+1,
\end{aligned}
$$

and adopting the convention $H_{j}^{*}()=.H_{j}\left(t, K^{*}(t), C^{*}(t), \lambda_{j}(t), t_{1}^{*}, \ldots, t_{n}^{*}\right)$, the maximum principle,

$$
\frac{\partial H_{j}^{*}(.)}{\partial C(t)} \equiv 0, \quad \forall t \in\left[t_{j-1}, t_{j}\right),
$$

and the canonical equations,

$$
\begin{aligned}
\dot{K}(t) & =\frac{\partial H_{j}^{*}(.)}{\partial \lambda_{j}(t)}, \quad \forall t \in\left[t_{j-1}, t_{j}\right), \\
\dot{\lambda}(t) & =-\frac{\partial H_{j}^{*}(.)}{\partial K(t)}, \quad \forall t \in\left[t_{j-1}, t_{j}\right], \quad j=1, \ldots, n+1 .
\end{aligned}
$$

are satisfied by the optimal trajectory. Finally, we have the matching conditions for the $j$ th regime switching $(j=1, \ldots, n)$,

$$
\begin{aligned}
& \left.H_{j}^{*}(.)\right|_{t_{j}^{*}}-\left.H_{j+1}^{*}(.)\right|_{t_{j}^{*}}+\left(\int_{t_{j-1}^{*}}^{t_{j}^{*}} \frac{\partial H_{j}^{*}(.)}{\partial t_{j}} \mathrm{~d} t+\int_{t_{j}^{*}}^{t_{j+1}^{*}} \frac{\partial H_{j+1}^{*}(.)}{\partial t_{j}} \mathrm{~d} t\right)=0 \quad \text { if } t_{j-1}^{*}<t_{j}^{*}<t_{j+1}^{*}, \\
& \left.H_{j}^{*}(.)\right|_{t_{j}^{*}}-\left.H_{j+1}^{*}(.)\right|_{t_{j}^{*}}+\left(\int_{t_{j-1}^{*}}^{t_{j}^{*}} \frac{\partial H_{j}^{*}(.)}{\partial t_{j}} \mathrm{~d} t+\int_{t_{j}^{*}}^{t_{j+1}^{*}} \frac{\partial H_{j+1}^{*}(.)}{\partial t_{j}} \mathrm{~d} t\right) \geqslant 0 \quad \text { if } t_{i-1}^{*}<t_{i}^{*}=t_{i+1}^{*},
\end{aligned}
$$




$$
\left.H_{j}^{*}(.)\right|_{t_{j}^{*}}-\left.H_{j+1}^{*}(.)\right|_{t_{j}^{*}}+\left(\int_{t_{j-1}^{*}}^{t_{j}^{*}} \frac{\partial H_{j}^{*}(.)}{\partial t_{j}} \mathrm{~d} t+\int_{t_{j}^{*}}^{t_{j+1}^{*}} \frac{\partial H_{j+1}^{*}(.)}{\partial t_{j}} \mathrm{~d} t\right) \leqslant 0 \quad \text { if } t_{i-1}^{*}=t_{i}^{*}<t_{i+1}^{*},
$$

and the following transversality condition:

$$
\lambda_{n+1}\left(t_{n+1}\right) \geqslant 0, \quad K\left(t_{n+1}\right) \geqslant 0, \quad \lambda_{n+1}\left(t_{n+1}\right) K\left(t_{n+1}\right)=0 .
$$

Proof

See Appendix.

The proof is based on the standard tools of variational calculus and considers independent variations in the state and the control variables [17-19]. In addition to the standard optimal control conditions, concerned with the choice of optimal consumption and capital between any two switches, we obtain the matching conditions that characterize the choice of the switching dates. Accordingly, the optimal decision must balance the benefit of upgrading the investment technology against the obsolescence and adjustment costs of such an upgrade.

\section{Remark 2}

The matching conditions for a two-stage problem (i.e. $n=1$ ) reduces to

$$
\left.H_{2}^{*}(.)\right|_{t_{1}^{*}}-\left.H_{1}^{*}(.)\right|_{t_{1}^{*}}=\int_{t_{0}}^{t_{1}^{*}} \frac{\partial H_{1}^{*}(.)}{\partial t_{1}} \mathrm{~d} t+\int_{t_{1}^{*}}^{t_{2}} \frac{\partial H_{2}^{*}(.)}{\partial t_{1}} \mathrm{~d} t \quad \text { if } t_{0}<t_{1}^{*}<t_{2},
$$

as stated in Tomiyama and Rossana [2].

Remark 3

In contrast with Tomiyama [1] and Makris [3], the Hamiltonians do not match at the switching instants because the state equation depends on the switching time explicitly. If this dependence is removed, it is clear that the matching conditions for the $k$ th regime switching reduces to

$$
\begin{array}{ll}
{\left[\left.H_{k}^{*}(.)\right|_{t_{k}^{*}}-\left.H_{k+1}^{*}(.)\right|_{t_{k}^{*}}\right]=0} & \text { if } t_{k-1}^{*}<t_{k}^{*}<t_{k+1}^{*}, \\
{\left[\left.H_{k}^{*}(.)\right|_{t_{k}^{*}}-\left.H_{k+1}^{*}(.)\right|_{t_{k}^{*}}\right] \geqslant 0} & \text { if } t_{k-1}^{*}<t_{k}^{*}=t_{k+1}^{*}, \\
{\left[\left.H_{k}^{*}(.)\right|_{t_{k}^{*}}-\left.H_{k+1}^{*}(.)\right|_{t_{k}^{*}}\right] \leqslant 0} & \text { if } t_{k-1}^{*}=t_{k}^{*}<t_{k+1}^{*},
\end{array}
$$

as stated in Makris [3] and with only a single regime switching (i.e. $n=1$ ), in Tomiyama [1].

The canonical conditions can be used to solve for $C$ and $K$ within each interval where the technology is held constant. Adopting a logarithmic utility function, from the resolution of these necessary conditions, we obtain:

$$
\begin{aligned}
C^{*}(t)= & \frac{\rho K\left(t_{j-1}\right)}{q\left(t_{j-1}\right)} \frac{\mathrm{e}^{-\frac{\eta}{\theta} q\left(t_{j-1}\right)\left(1-\mathrm{e}^{-\theta\left(t-t_{j-1}\right)}-A\left(t-t_{j-1}\right) \frac{\theta}{\eta}\right)}}{\mathrm{e}^{\rho\left(t-t_{j-1}\right)}\left(1-\mathrm{e}^{-\rho\left(T-t_{j-1}\right)}\right)}, \quad \forall t \in\left[t_{j-1}, t_{j}\right), \\
K^{*}(t)= & K\left(t_{j-1}\right)\left(\frac{1-\mathrm{e}^{-\rho(t-T)}}{1-\mathrm{e}^{-\rho\left(t_{j-1}-T\right)}}\right) \mathrm{e}^{-\frac{\eta}{\theta} q\left(t_{j-1}\right)\left(1-\mathrm{e}^{-\theta\left(t-t_{j-1}\right)}-A\left(t-t_{j-1}\right) \frac{\theta}{\eta}\right)}, \\
& \forall t \in\left[t_{j-1}, t_{j}\right), \quad j=1,2, \ldots, n+1 .
\end{aligned}
$$

Notice that the consumption path is discontinuous at $t_{i}^{*}, i=1,2, \ldots, n$. Since $q\left(t_{i}\right)>q\left(t_{i-1}\right)$, we have the level of consumption along the old technology regime is higher than in the new regime in each of the technology switches. This stems from the fact that a higher level of embodied technical change is associated with a decrease in the relative price of capital, which increases the user cost of capital by the so-called obsolescence costs.

When switching to a new technology, an improvement in the efficiency of capital goods, referred to as growth rate advantage, will be experienced. The matching conditions given in (11)-(13) 
will solve the trade-off between this growth rate advantage and the adjustment cost of technology adoption together with obsolescence cost inherent to embodiment. The consecutive stages of our problem are connected through the continuity condition (7). The value of the co-state variable at the instant of switching to a new technology represents the rate of change of the optimal value function with respect to the change in the capital stock. Accordingly, $\lambda_{i+1}\left(t_{i}^{*}\right)-\lambda_{i}\left(t_{i}^{*}\right)$ represents the marginal gain in utility terms of an increase in the capital stock at the $i$ th technology switching instant.

Having established the optimality conditions for the multi-stage optimal control problem for a given number of switches, now we turn our attention to the optimal number of switches within a given planning horizon.

\subsection{Optimal number of technology adoptions}

The matching conditions can be used to analyze the corner solutions (possibility and the economic meaning) in the optimal timing of technology upgrades, i.e. immediate switching and technological sclerosis. In general, in such a system with $n$ switches, one has to consider $2^{n+1}-1$ possible corner solution cases. Among these, if optimal path follows $t_{0}<t_{1}^{*}=t_{2}^{*}=\cdots=t_{n}^{*}=T$, then the agent faces a sclerosis at the initial technology so that no technology adoption actually occurs. If the optimal path follows $t_{0}<t_{1}^{*}<t_{2}^{*}=\cdots=t_{n}^{*}=T$, then it is clear that once the agent switches to the new technology at $t_{1}^{*}$, it sticks to that till the end of the planning horizon so that the optimal number of technology adoption equals one.

In accordance with these, given a sufficiently large maximum number of technology upgrades $n$, the necessary conditions for $t_{0}<t_{1}^{*}<t_{2}^{*}<\cdots<t_{m}^{*}<t_{m+1}^{*}=\cdots=t_{n}^{*}=t_{n+1}$ can easily be written in reference to (11)-(13), in which the optimal number of technology adoptions actually equals to $m$. Then, the optimal number of switches $m^{*}$ can be determined as a solution to

$$
\underset{m \in\{0,1,2, \ldots, n\}}{\arg \max }\left\{W\left(K^{*}(t), C^{*}(t), t_{1}^{*}, \ldots, t_{n}^{*}\right) \mid \begin{array}{ll}
t_{j}^{*}<t_{j+1}^{*}, & j=0, \ldots, m, \\
t_{j}^{*}=t_{j+1}^{*}, & j=m+1, \ldots, n .
\end{array}\right\}
$$

Alternatively, concentrating only on the interior solutions for the optimal timing of technology upgrades, the optimal number of technology adoptions can be determined as a solution to

$$
\underset{n \in\{0,1,2,3, \ldots\}}{\arg \max }\left\{W\left(K^{*}(t), C^{*}(t), t_{1}^{*}, \ldots, t_{n}^{*}\right) \mid t_{0}<t_{1}^{*}<t_{2}^{*}<\cdots<t_{n}^{*}<t_{n+1}\right\} .
$$

As we are not able to derive the closed form representation of the optimal number of switches and thus, the optimal timing of technology adoptions, we resort to numerical analysis."

\section{NUMERICAL ANALYSIS}

In this section, we illustrate the properties of the solution to the multi-stage optimal control problem. We study to which extent embodiment matters in the optimal pace of technology adoption. We analyze how the trade-off, which exists between growth rate advantage and the obsolescence and adjustment costs, shift in response to an exogenous change in the parameters of the model with a special emphasis on the planning horizon. To this end, we adopt a logarithmic utility function and summarize the benchmark parameterization of the model in Table I.

In a competitive equilibrium the relative price of capital goods would be given by $1 / q$, since this shows how much output or consumption goods must be given up in order to purchase a new unit of equipment. Accordingly, using the decline rate of the relative price of equipment as a proxy, several studies attempt to measure the rate of investment-specific technological progress and evaluate its role in predicting recent and prospective productivity growth trends of the US ISee Boucekkine et al. [12], in which an analytical computation of optimal timing of a single technology adoption
is presented via two-stage optimal control. 
Table I. Values of the parameters as a benchmark.

\begin{tabular}{lcccccc}
\hline$\gamma$ & $\rho$ & $\theta$ & $\eta$ & $A$ & $K_{0}$ & $T$ \\
\hline 0.02 & 0.03 & 0.7 & 1 & 2 & 1 & 30 \\
\hline
\end{tabular}

Table II. Determination of the optimal number of adoptions.

\begin{tabular}{rrrrrc}
\hline$m$ & $t_{1}^{*}$ & $t_{2}^{*}$ & $t_{3}^{*}$ & $t_{4}^{*}$ & $W^{*}$ \\
\hline 1 & 11.32 & & & & 437.711 \\
$\mathbf{2}$ & $\mathbf{8 . 9 9}$ & $\mathbf{1 8 . 1 1}$ & & & $\mathbf{4 4 0 . 3 3 6}$ \\
3 & 8.99 & 18.11 & 30.00 & & 440.336 \\
4 & 8.99 & 18.11 & 30.00 & 30.00 & 440.336 \\
\hline
\end{tabular}

economy (see, for the review of the concepts and recent estimates, [20]). Among those, Del Rio [6], Pakko [20] and Cummins and Violante [21], using different assumptions and methodologies, find that the investment-specific technological progress amounts to $2.27,1.86$ and $1.50 \%$, respectively. In accordance with these, we set $\gamma$ to 0.02 .

There is vast number of studies noting that the productivity may not be high at the early stages of the implementation of the new technique until a sufficient level of experience is reached.** Jovanovic and Nyarko [15] show that increases in the productivity of individual plants following their start-ups are typically large and realized in a short period of time. Bahk and Gort [22], using a panel data of new plants from 15 different industries, estimate that adjustment to the new technology is realized within 6 years and at each year $1 \%$ increase is associated with the increase in the output. Consistent with these typical learning-by-doing models, we assume that the adjustment cost of technology adoption is not permanent and it is asymptotically eliminated with a speed of $\theta=0.7$.

Obsolescence cost inherent to embodiment and the accelerated erosion of physical capital following a technology adoption rule out the continuous adoption of new technologies within a finite planning horizon. Under the parameter values presented in Table I, determination of the optimal number of technology upgrades is summarized in Table II. We set the maximum number of possible technology upgrades to 4 (i.e. $n=4)$. In case of a single switch $(m=1)$, the optimal timing of adoption is 11.32 and the maximum lifetime utility level that can been attained is 437.711 . Allowing for two switches, we obtain that the resulting optimal lifetime utility level is 440.336 . When we analyze the case of three or more technology switches, the resulting improvement in the efficiency of investment is not enough to cover the obsolescence and adjustment costs under the given planning horizon so that we obtain corner solutions with $t_{3}^{*}=t_{4}^{*}=T$ and obviously no improvement in the lifetime utility level. Thus, for our benchmark setting, the optimal plan incorporates actually two technology upgrades with $t_{1}^{*}=8.99$, and $t_{2}^{*}=18.11$, Accordingly, the initial technology with $q\left(t_{0}\right)$ will be used for 8.99 years and then replaced by the second technology with $q\left(t_{1}^{*}\right)$. The second technology will be in use for 9.12 years and then replaced by the final technology with $q\left(t_{2}^{*}\right)$.

When we analyze the behavior of the adoption pattern with respect to time discounting, we observe that a higher impatience rate tends to delay adoption. Indeed, if the agent is impatient enough, even a decrease in the optimal number of adoptions may occur. The effect of the time discounting on the pattern of technology adoptions is summarized in Table III. The delay in the adoption of the more advanced technologies as $\rho$ increases is due to the increases in the obsolescence costs. However, there may exist an opposing effect that tends to accelerate adoption. Since the sooner the adoption, the sooner the accelerated erosion of the preexisting physical capital will occur and the sooner the best productivity level of the new technology will be reached,

\footnotetext{
**For a general survey, see Greenwood and Jovanovic [16].
} 
Table III. The effect of changes in $\rho$.

\begin{tabular}{lcccr}
\hline$\rho$ & $m^{*}$ & $t_{1}^{*}$ & $t_{2}^{*}$ & $W^{*}$ \\
\hline 0.01 & 2 & 8.88 & 17.93 & 661.991 \\
0.02 & 2 & 8.93 & 18.01 & 538.665 \\
$\mathbf{0 . 0 3}$ & $\mathbf{2}$ & $\mathbf{8 . 9 9}$ & $\mathbf{1 8 . 1 1}$ & $\mathbf{4 4 0 . 3 3 6}$ \\
0.04 & 2 & 9.06 & 18.22 & 361.677 \\
0.05 & 2 & 9.14 & 18.35 & 298.535 \\
0.20 & 1 & 11.42 & & 31.020 \\
\hline
\end{tabular}

Table IV. The effect of changes in $\gamma$.

\begin{tabular}{lrrrrrc}
\hline$\gamma$ & $m^{*}$ & \multicolumn{1}{c}{$t_{1}^{*}$} & $t_{2}^{*}$ & $t_{3}^{*}$ & $t_{4}^{*}$ & $W^{*}$ \\
\hline 0.010 & 1 & 13.08 & & & & 417.817 \\
$\mathbf{0 . 0 2 0}$ & $\mathbf{2}$ & $\mathbf{8 . 9 9}$ & $\mathbf{1 8 . 1 1}$ & & & $\mathbf{4 4 0 . 3 3 6}$ \\
0.025 & 2 & 8.43 & 17.18 & & & 455.464 \\
0.030 & 3 & 7.10 & 14.22 & 21.37 & & 472.624 \\
0.040 & 3 & 6.53 & 13.15 & 20.02 & & 514.134 \\
0.050 & 4 & 5.50 & 11.02 & 16.58 & 22.27 & 565.326 \\
\hline
\end{tabular}

Table V. The effect of changes in $\theta$.

\begin{tabular}{lrrrrrc}
\hline$\theta$ & $m^{*}$ & $t_{1}^{*}$ & $t_{2}^{*}$ & $t_{3}^{*}$ & $t_{4}^{*}$ & $W^{*}$ \\
\hline 0.1 & 0 & & & & & 361.782 \\
0.3 & 1 & 13.42 & & & & 392.810 \\
0.5 & 1 & 11.95 & & & & 422.704 \\
$\mathbf{0 . 7}$ & $\mathbf{2}$ & $\mathbf{8 . 9 9}$ & $\mathbf{1 8 . 1 1}$ & & & $\mathbf{4 4 0 . 3 3 6}$ \\
0.9 & 2 & 8.36 & 17.06 & & & 451.819 \\
1.1 & 3 & 7.05 & 14.12 & 21.22 & & 459.994 \\
1.3 & 3 & 6.65 & 13.37 & 20.26 & & 466.602 \\
1.5 & 3 & 6.37 & 12.87 & 19.66 & & 471.650 \\
1.7 & 4 & 5.65 & 11.31 & 16.98 & 22.71 & 475.780 \\
\hline
\end{tabular}

increasing the growth rate advantage of a technology upgrade (see, for an analytical inspection in a single technology adoption problem, [12]). With our parameter values, as $\rho$ increases, the obsolescence cost clearly dominates the growth rate advantage.

In contrast with the effect of an increase in the impatience rate, an increase in the investmentspecific technological progress leads to an acceleration in the adoption of the new technologies. Table IV presents the effect of a change in $\gamma$ on the optimal pattern of technology adoptions. This is far from surprising because of the associated increase in the growth rate advantage inherent to the adoption of the new technologies. This reduces the time required for the growth rate advantage to dominate costs due to obsolescence and the accelerated depreciation so that an acceleration in the adoption of the new technologies will occur. Accordingly, an increase in the rate of investment-specific technological progress that does not alter the optimal number of switches reduces the lifetimes of the technologies in use except the final technology. The lifetime devoted to the use of the final technology will be longer as the duration left after the last switch will not be sufficient for covering the obsolescence and adjustment costs of a further switch. However, a sufficient increase in $\gamma$ would also increase the optimal number of adoptions within a given planning horizon. This leads to a further decrease in the duration between two consecutive adoptions.

Table V reports the optimal pattern of technology adoptions for different values of $\theta$. An increase in $\theta$ accelerates the adoption so that the duration between two consecutive adoptions would decrease. In other words, it tends to decrease the lifetimes of the early adopted technologies and 
Table VI. The effect of changes in $T$.

\begin{tabular}{ccccccc}
\hline$T$ & $m^{*}$ & $t_{1}^{*}$ & $t_{2}^{*}$ & $t_{3}^{*}$ & $t_{4}^{*}$ & $W^{*}$ \\
\hline 15 & 0 & & & & & 119.734 \\
20 & 1 & 8.94 & & & & 209.481 \\
25 & 1 & 9.99 & & & & 317.741 \\
$\mathbf{3 0}$ & $\mathbf{2}$ & $\mathbf{8 . 9 9}$ & $\mathbf{1 8 . 1 1}$ & & & $\mathbf{4 4 0 . 3 3 6}$ \\
35 & 2 & 9.64 & 19.73 & & & 573.700 \\
40 & 3 & 9.03 & 18.13 & 27.42 & & 715.885 \\
45 & 3 & 9.51 & 19.23 & 29.43 & & 863.090 \\
50 & 4 & 9.06 & 18.16 & 27.37 & 36.82 & 1015.21 \\
\hline
\end{tabular}

increase the lifetime of the final technology to be used. Moreover, a sufficient increase in the speed of adjustment may lead to an increase in the optimal number of adoptions. In such cases, the optimal plan features a further decrease in the duration between two consecutive technology adoptions. On the other hand, for sufficiently low values of $\theta$, the agent would never switch to a better technology and face a technological sclerosis.

Finally, we will consider how the optimal pattern of technology adoptions shift in response to the changes in the planning horizon. It is clear that a longer planning horizon provides an incentive to delay adoptions in order to benefit more from the growth rate advantage. In Boucekkine et al. [12], considering a single technology adoption problem, it is proven that a longer time horizon leads to a delay in the adoption of the new technology. Consistent with these, when we consider the effect of an increase in $T$ that does not alter the optimal number of switches, we note that the adoption of new technologies are delayed and the lifetimes of each technology in use are increased. However, such an increase in the planning horizon may also lead to an increase in the optimal number of adoptions, which would clearly reduce the lifetimes of each technology in use as well. The effects of changes in $T$ on the optimal number of switches and hence, on the dates when these switches will occur, are presented in Table VI.

With our benchmark parameterization, increasing the planning horizon to 35 years does not alter the optimal number of technology adoptions and leads to a delay in the adoption of the two technologies. As the optimal number of technology adoptions is left unchanged, such an increase in the planning horizon creates an 'anticipation effect' in the sense that knowing better technologies will appear in the future makes the postponement of the adoptions optimal. Accordingly, the lifetimes of all the technologies in use increase. However, increasing further the planning horizon to 40 years increases the optimal number technology adoptions so that the technology would be updated three times incorporating shorter lifetimes for each. On the other hand, if the planning horizon were short enough $(T \leqslant 15)$, the agent would stick to the initial technology and end up with a technological sclerosis.

If the horizon were infinite, then the optimal plan would incorporate infinite number of technology upgrades. In such an optimal growth framework, Parente [9] and Iacompetta [23] analyze the continuous adoption of new technologies and conjecture that the optimal lifetime of a technology would be constant through time $e^{\dagger \dagger}$. However, a finite planning horizon leads us to obtain nonstationary durations between consecutive adoptions. As the final technology has no better subsequent technology to compete with, it should not be surprising that the agent stays around it longer than the rest. Indeed, we show that the lifetimes of the technologies in use follow a

\footnotetext{
${ }^{\dagger}$ When analyzing the optimal machine replacement policy of firms, such an equidistance property has also been assumed by Terborgh [24] and Smith [25]. Malcolmson [26] has derived two sequences that bound the optimal machine life. Assuming that the holding cost of a machine is constant and adopting a linear rate of technological progress and deterioration, Van Hilten [27] has shown that these sequences converge to the same limit so that the optimal machine life is constant through time. However, this convergence has not yet been confirmed or denied analytically in general. With an exponentially growing technology frontier and non-linear utility, there is no room for showing, even numerically, the equidistance property in our model.
} 
monotonically increasing path and the admissible plans that incorporate more frequent technology switches through the end of the planning horizon are not optimal.

In addition to the eventual adjustment costs associated with technology adoption, the crucial factor in these is the obsolescence cost inherent to the embodiment. The gain in the rate of embodied technological change is associated with a reduction in the relative price of capital so that the consumption should drop from the date of each switch. Moreover, it is important to note that switching to more embodiment implies more drop in the level of consumption. Because of this, as agent grows older, even though the increasing growth rate advantage creates an incentive for more frequent switching, the associated increase in the obsolescence cost prohibits to do so. Therefore, it turns out to be optimal to devote more time for using later technologies as they will be endowed with a higher level of investment efficiency and eventually provide a higher level of consumption.

\section{CONCLUSION}

In this paper, we provide a multi-stage optimal control approach for analyzing the optimal pattern of technology adoptions under a finite planning horizon. To do so, we have derived the necessary conditions of optimality for such multi-stage optimal control problems in which the adjustable switching times appear as an argument of the state equation. We have provided numerically the effects of the planning horizon, growth rate of technology, discounting parameter and the speed of adjustment to the new technology on the optimal pattern of technology adoptions.

We believe that this model provides a rich structure that could be used to examine several other types of optimal choice and timing problems. On growth-related issues, a decentralized equilibria could be considered in order to analyze the technological leapfrogging among firms and industries. It could be extended to analyze the effects of capital market efficiency, the role of uncertainty and the market structure on technology adoption. These are in our research agenda.

\section{APPENDIX A}

\section{Proof}

To derive necessary conditions for the optimum, we utilize the standard tools of variational calculus (see $[17,19,18]$ ). To this end, we define the modified performance index, using the notion of Lagrange multipliers. Associating continuous and piecewise continuously differentiable real-valued functions, $\lambda_{j}(t), \forall t \in\left[t_{j-1}, t_{j}\right], j=1,2, \ldots, n+1$, with each equation of (2), we have:

$$
\begin{aligned}
W\left(K(t), C(t), t_{1}, \ldots, t_{n}\right)= & \sum_{j=1}^{n+1} \int_{t_{j-1}}^{t_{j}}\left[u(C(t)) \mathrm{e}^{-\rho t}\right. \\
& \left.+\lambda_{j}(t)\left(q\left(t_{j-1}\right)\left((A K(t)-C(t))-\eta \mathrm{e}^{-\theta\left(t-t_{j-1}\right)} K(t)\right)-\dot{K}(t)\right)\right] \mathrm{d} t .
\end{aligned}
$$

Defining the Hamiltonian function for each stage by means of a Legendre transformation,

$$
\begin{gathered}
H_{j}\left(t, K(t), C(t), \lambda_{j}(t), t_{j-1}, t_{j}\right)=u(C(t)) \mathrm{e}^{-\rho t}+\lambda_{j}(t) q\left(t_{j-1}\right)\left[(A K(t)-C(t))-\eta \mathrm{e}^{-\theta\left(t-t_{j-1}\right)} K(t)\right], \\
\forall t \in\left[t_{j-1}, t_{j}\right), \quad j=1, \ldots, n+1,
\end{gathered}
$$

we obtain:

$$
W\left(t, K(t), C(t), t_{1}, \ldots, t_{n}\right)=\sum_{j=1}^{n+1} \int_{t_{j-1}}^{t_{j}}\left[H_{j}\left(t, K(t), C(t), \lambda_{j}(t), t_{j-1}, t_{j}\right)-\lambda_{j}(t) \dot{K}(t)\right] \mathrm{d} t .
$$

\footnotetext{
$\$$ Mateos-Planas [11] considers the life-cycle technology adoption problem of an agent operating a single firm that produces output using one machine and predicts also non-stationarity in the sense that an optimal plan may incorporate both types of adopted technologies that are to be learned and that are to be skipped without learning. However, as it leaves no room to deal with capital accumulation and adopts a step-wise discrete learning process, concludes that the adoptions may become more frequent as the agent grows older.
} 
Integrating the last term of this equation by parts, we have

$$
\sum_{j=1}^{n+1} \int_{t_{j-1}}^{t_{j}} \lambda_{j}(t) \dot{K}(t) \mathrm{d} t=\sum_{j=1}^{n+1}\left(\lambda_{j}\left(t_{j}\right) K\left(t_{j}\right)-\lambda_{j}\left(t_{j-1}\right) K\left(t_{j-1}\right)\right)-\sum_{j=1}^{n+1} \int_{t_{j-1}}^{t_{j}} \dot{\lambda}_{j}(t) K(t) \mathrm{d} t .
$$

This immediately implies that

$$
\begin{aligned}
W\left(t, K(t), C(t), t_{1}, t_{2}, \ldots, t_{n}\right)= & \sum_{j=1}^{n+1}\left(-\lambda_{j}\left(t_{j}\right) K\left(t_{j}\right)+\lambda_{j}\left(t_{j-1}\right) K\left(t_{j-1}\right)\right) \\
& +\sum_{j=1}^{n+1} \int_{t_{j-1}}^{t_{j}}\left[H_{j}\left(t, K(t), C(t), \lambda_{j}(t), t_{j-1}, t_{j}\right)+\dot{\lambda}_{j}(t) K(t)\right] \mathrm{d} t .
\end{aligned}
$$

Let the path $\left(K^{*}(t), C^{*}(t), t_{1}^{*}, \ldots, t_{n}^{*}\right)$ be optimal. Recall that along the optimal path, $C^{*}(t)=$ $Z(t) K^{*}(t)-\left(\dot{K}^{*}(t)\right) /\left(q\left(t_{j-1}^{*}\right)\right)$, where $Z(t)=\left(A-\eta \mathrm{e}^{-\theta\left(t-t_{j-1}^{*}\right)}\right), \quad \forall t \in\left[t_{j-1}^{*}, t_{j}^{*}\right), j=1, \ldots, n+1$. Note that $Z(t)$ is continuous on each $\left[t_{j-1}^{*}, t_{j}^{*}\right), j=1, \ldots, n+1$. Following from Askenazy and Le Van [28], since $K^{*}(t)$ is continuous, $\dot{K}^{*}(t)$ is piecewise continuous, and $u^{\prime}(0)=+\infty$, we have $C^{*}(t)>0, \forall t \in\left[t_{j-1}^{*}, t_{j}^{*}\right), j=1, \ldots, n+1$. This clearly implies that $C^{*}(t)>0, K^{*}(t)>0, \forall t \in[0, T)$.

We will now consider independent variations of $\delta C(t), \delta K(t)$ and $\delta t_{j}, j=1,2, \ldots, n+1$, and generate the following neighboring admissible paths

$$
\begin{aligned}
C(t, \varepsilon) & \equiv C^{*}(t)+\varepsilon \delta C(t), \\
K(t, \varepsilon) & \equiv K^{*}(t)+\varepsilon \delta K(t), \\
t_{j}(\varepsilon) & \equiv t_{j}^{*}+\varepsilon \delta t_{j}, \quad j=1,2, \ldots, n,
\end{aligned}
$$

by varying a scalar $\varepsilon \in \mathbb{R}$. In accordance with these,

$$
\begin{aligned}
\mathscr{W}(\varepsilon) \equiv & W\left(K(t, \varepsilon), C(t, \varepsilon), t_{1}(\varepsilon), \ldots, t_{n}(\varepsilon)\right) \\
= & \sum_{j=1}^{n+1} \int_{t_{j-1}(\varepsilon)}^{t_{j}(\varepsilon)}\left[H_{j}\left(t, K(t, \varepsilon), C(t, \varepsilon), \lambda_{j}(t), t_{j-1}(\varepsilon), t_{j}(\varepsilon)\right)+\dot{\lambda}_{j}(t) K(t, \varepsilon)\right] \mathrm{d} t \\
& +\sum_{j=1}^{n+1}\left(-\lambda_{j}\left(t_{j}(\varepsilon)\right) K\left(t_{j}(\varepsilon), \varepsilon\right)+\lambda_{j}\left(t_{j-1}(\varepsilon)\right) K\left(t_{j-1}(\varepsilon), \varepsilon\right)\right) .
\end{aligned}
$$

It is clear that $\mathscr{W}(\varepsilon) \leqslant \mathscr{W}(0), \forall \varepsilon \in \mathbb{R}$, as $\left(K^{*}(t), C^{*}(t), t_{1}^{*}, \ldots, t_{n}^{*}\right)$ is optimal. Remember that $K^{*}(t)=K(t, \varepsilon=0)$ refers to the path of the state variable corresponding to the optimal plan $\left(C^{*}(t), t_{1}^{*}, \ldots, t_{n}^{*}\right)$. The optimality then requires that $\mathscr{W}^{\prime}(0) \equiv 0$, for all independent variations $\delta C$, $\delta K$, and $\delta t_{j}, j=1,2, \ldots, n$, Accordingly, differentiating $\mathscr{W}(\varepsilon)$ with respect to $\varepsilon$ by utilizing Leibniz's rule, we obtain:

$$
\begin{aligned}
\mathscr{W}^{\prime}(\varepsilon)= & \sum_{j=1}^{n+1}\left(\left.H_{j}(.)\right|_{t_{j}(\varepsilon)}\right) \delta t_{j}-\sum_{j=1}^{n+1}\left(\left.H_{j}(.)\right|_{t_{j-1}(\varepsilon)}\right) \delta t_{j-1} \\
& +\sum_{j=1}^{n+1} \int_{t_{j-1}(\varepsilon)}^{t_{j}(\varepsilon)}\left[\left(\frac{\partial H_{j}(.)}{\partial K}+\dot{\lambda}_{j}(t)\right) \delta K(t)+\left(\frac{\partial H_{j}(.)}{\partial C}\right) \delta C(t)\right] \mathrm{d} t \\
& +\sum_{j=1}^{n+1} \int_{t_{j-1}(\varepsilon)}^{t_{j}(\varepsilon)}\left[\left(\frac{\partial H_{j}(.)}{\partial t_{j}}\right) \delta t_{j}+\left(\frac{\partial H_{j}(.)}{\delta t_{j-1}}\right) \delta t_{j-1}\right] \mathrm{d} t+\sum_{j=1}^{n+1} \lambda_{j}\left(t_{j-1}(\varepsilon)\right)\left(\dot{K}_{j-1}\left(t_{j-1}(\varepsilon), \varepsilon\right) \delta t_{j-1}\right. \\
& \left.+\delta K\left(t_{j-1}\right)\right)-\sum_{j=1}^{n+1} \lambda_{j}\left(t_{j}(\varepsilon)\right)\left(\dot{K}\left(t_{j}(\varepsilon), \varepsilon\right) \delta t_{j}+\delta K\left(t_{j}\right)\right) .
\end{aligned}
$$


As $\left\{t_{0}, K\left(t_{0}\right)\right\}$ and $T$ are the given parameters of the model, setting $\delta t_{0}, \delta K\left(t_{0}\right)$ and $\delta t_{n+1}(\delta T)$ equal to zero and evaluating the derivative in (A3) at $\varepsilon=0$, we have:

$$
\begin{aligned}
\mathscr{W}^{\prime}(0)= & \sum_{j=1}^{n+1} \int_{t_{j-1}^{*}}^{t_{j}^{*}}\left[\left(\frac{\partial H_{j}^{*}(.)}{\partial K}+\dot{\lambda}_{j}(t)\right) \delta K(t)+\frac{\partial H_{j}^{*}(.)}{\partial C} \delta C(t)\right] \mathrm{d} t \\
& +\sum_{j=1}^{n}\left[\left.H_{j}^{*}(.)\right|_{t_{j}^{*}}-\left.H_{j+1}^{*}(.)\right|_{t_{j}^{*}}+\left(\int_{t_{j-1}^{*}}^{t_{j}^{*}} \frac{\partial H_{j}^{*}(.)}{\partial t_{j}} d t+\int_{t_{j}^{*}}^{t_{j+1}^{*}} \frac{\partial H_{j+1}^{*}(.)}{\partial t_{j}} \mathrm{~d} t\right)\right] \delta t_{j} \\
& +\sum_{j=1}^{n}\left(\lambda_{j+1}\left(t_{j}^{*}\right)-\lambda_{j}\left(t_{j}^{*}\right)\right)\left[\dot{K}\left(t_{j}\right) \delta t_{j}+\delta K\left(t_{j}\right)\right]-\lambda_{n+1}\left(t_{n+1}\right) \delta K\left(t_{n+1}\right),
\end{aligned}
$$

where $H_{j}^{*}()=.H_{j}\left(t, K^{*}(t), C^{*}(t), \lambda_{j}(t), t_{j-1}^{*}, t_{j}^{*}\right)$,

Using the notion of Lagrange multipliers, the functions $\lambda_{j}($.$) has to be chosen appropriately in$ order to satisfy $\partial H_{j}^{*}(.) / \partial C=0, \forall t \in\left[t_{j-1}, t_{j}\right)$, Hence,

$$
\sum_{j=1}^{n+1} \int_{t_{j-1}^{*}}^{t_{j}^{*}}\left(\frac{\partial H_{j}^{*}(.)}{\partial C} \delta C(t)\right) \mathrm{d} t=0, \quad \forall \delta C(t) .
$$

Accordingly, noting that $\delta C(t)$ and $\delta K(t)$ are arbitrary, we have

$$
\dot{\lambda}_{j}(t)=-\frac{\partial H_{j}^{*}(.)}{\partial K}, \quad \forall t \in\left[t_{j-1}, t_{j}\right], \quad j=1, \ldots, n+1 .
$$

Since $\left\{t_{j}, K\left(t_{j}\right)\right\}, j=1,2, \ldots, n$, are free, we obtain continuity and matching conditions that characterize the optimal timing of technology adoptions. The continuity conditions,

$$
\lambda_{j+1}\left(t_{j}^{*}\right)=\lambda_{j}\left(t_{j}^{*}\right), \quad j=1,2, \ldots, n,
$$

follow easily from the third row of (A4). As for the matching conditions, from the second row of (A4), whenever the optimal path includes $t_{j-1}^{*}<t_{j}^{*}<t_{j+1}^{*}$, we have

$$
\left.H_{j}^{*}(.)\right|_{t_{j}^{*}}-\left.H_{j+1}^{*}(.)\right|_{t_{j}^{*}}+\left(\int_{t_{j-1}^{*}}^{t_{j}^{*}} \frac{\partial H_{j}^{*}(.)}{\partial t_{j}} \mathrm{~d} t+\int_{t_{j}^{*}}^{t_{j+1}^{*}} \frac{\partial H_{j+1}^{*}(.)}{\partial t_{j}} \mathrm{~d} t\right)=0,
$$

for the $j$ th technology switching. If, instead, the optimal path is such that $t_{j-1}^{*}<t_{j}^{*}=t_{j+1}^{*}$, then the admissible neighboring paths involve either the same or an earlier termination time than $t_{j+1}^{*}$. Assuming that $\delta t_{j}>0$, such a requirement would impose that $\varepsilon \leqslant 0$. Then, by Kuhn-Tucker conditions, the optimality condition $\mathscr{W}^{\prime}(0)=0$ would be altered to $\mathscr{W}^{\prime}(0) \geqslant 0$ implying that

$$
\left.H_{j}^{*}(.)\right|_{t_{j}^{*}}-\left.H_{j+1}^{*}(.)\right|_{t_{j}^{*}}+\left(\int_{t_{j-1}^{*}}^{t_{j}^{*}} \frac{\partial H_{j}^{*}(.)}{\partial t_{j}} \mathrm{~d} t+\int_{t_{j}^{*}}^{t_{j+1}^{*}} \frac{\partial H_{j+1}^{*}(.)}{\partial t_{j}} \mathrm{~d} t\right) \geqslant 0 .
$$

Similarly, if the optimal path involves $t_{j-1}^{*}=t_{j}^{*}<t_{j+1}^{*}$, then we have

$$
\left.H_{j}^{*}(.)\right|_{t_{j}^{*}}-\left.H_{j+1}^{*}(.)\right|_{t_{j}^{*}}+\left(\int_{t_{j-1}^{*}}^{t_{j}^{*}} \frac{\partial H_{j}^{*}(.)}{\partial t_{j}} \mathrm{~d} t+\int_{t_{j}^{*}}^{t_{j+1}^{*}} \frac{\partial H_{j+1}^{*}(.)}{\partial t_{j}} \mathrm{~d} t\right) \leqslant 0,
$$

as a necessary condition of optimality.

Finally, we have to consider the terminal condition. The level of the state is free, but only subject to $K(t) \geqslant 0$. Following Chiang [18], we need to consider two possible cases in the optimal solution. If $K^{*}(t)>0$, then the transversality condition $\lambda_{n+1}\left(t_{n+1}\right)=0$ follows directly from the fourth row of (A4). If, on the other hand, the terminal condition is binding so that $K^{*}(t)=0$, then the admissible neighboring paths should satisfy $K(t) \geqslant 0$. Such a requirement imposes that $\varepsilon \geqslant 0$ 
$[\varepsilon \leqslant 0]$ whenever $\delta K(t)>0[\delta K(t)<0]$. Then, by Kuhn-Tucker conditions, we have the transversality condition, $\lambda_{n+1}\left(t_{n+1}\right) \geqslant 0$ for $K^{*}(t)=0$. Combining these two possible cases, the transversality condition can be written as: $\lambda_{n+1}\left(t_{n+1}\right) \geqslant 0, K\left(t_{n+1}\right) \geqslant 0, K\left(t_{n+1}\right) \lambda_{n+1}\left(t_{n+1}\right)=0$.

\section{REFERENCES}

1. Tomiyama K. Two-stage optimal control problems and optimality conditions. Journal of Economic Dynamics and Control 1985; 9:317-337.

2. Tomiyama K, Rossana R. Two-stage optimal control problems with an explicit switch point dependence: optimality criteria and an example of delivery lags and investment. Journal of Economic Dynamics and Control 1989; 13:319-337.

3. Makris M. Necessary conditions for infinite horizon discounted two-stage optimal control problems. Journal of Economic Dynamics and Control 2001; 25:1935-1950.

4. Boucekkine R, del Río F, Licandro O. Embodied technological change, learning by doing and the productivity slowdown. Scandinavian Journal of Economics 2003; 105(1):87-93.

5. Krusell P. Investment-specific R\&D and the decline in the relative price of capital. Journal of Economic Growth 1998; 3:131-141.

6. del Río F. Neutral, investment-specific technical progress the productivity slowdown. Recherches économiques de Louvain 2002 68(1-2):37-55.

7. Boedo HJM. Optimal technology and development. Journal of Macroeconomics 2009; DOI: 10.1016/ j.jmacro.2009.07.003.

8. Boucekkine R, del Río F, Martinez B. Technological progress, obsolescence, and depreciation. Oxford Economic Papers 2009; 61:440-466.

9. Parente S. Technology adoption, learning by doing, and economic growth. Journal of Economic Theory 1994; 63:346-369.

10. Parente S. Learning-by-using the switch to better machines. Review of Economic Dynamics 2000 3:675-703.

11. Mateos-Planas X. Technology adoption with finite horizons. Journal of Economic Dynamics and Control 2004; 28:2129-2154.

12. Boucekkine R, Saglam C, Vallée T. Technology adoption under embodiment: a two-stage optimal control approach. Macroeconomic Dynamics 2004; 8(2):250-271.

13. Solow R. Investment and technological progress. In Mathematical Methods in the Social Sciences 1959, Kenneth Arrow J, Karlin S, Suppes P (eds). Stanford University Press: Stanford, CA, 1960; 89-104.

14. Greenwood J, Hercowitz Z, Krusell P. Long-run implications of investment-specific technological change. American Economic Review 1997; 87:342-362.

15. Jovanovic B, Nyarko Y. Learning by doing and the choice of technology. Econometrica 1996; 64:1299-1310.

16. Greenwood J, Jovanovic B. Accounting for Growth. In New Directions in Productivity Analysis, NBER Studies in Income and Wealth, Dean E, Harper M, Hulten C (eds), vol. 63. Chicago University Press: Chicago, 2001.

17. Bryson AH, Ho Y. Applied Optimal Control: Optimization, Estimation, and Control. Hemisphere Publishing: New York, 1975.

18. Chiang AC. Elements of Dynamic Optimization. McGraw-Hill: New York, 1992.

19. Kamien MI, Schwartz NL. Dynamic Optimization. North-Holland: Amsterdam, 1991.

20. Pakko MR. Investment-specific technology growth: concepts and recent estimates. Federal Reserve Bank of St. Louis Review 2002; 84:37-48.

21. Cummins JG, Violante GL. Investment specific technical change in the united states (1947-2000): measurement and applications. Review of Economic Dynamics 2002; 5(2):243-284.

22. Bahk B, Gort M. Decomposing learning by doing in new plants. Journal of Political Economy 1993; 101(4): 561-583.

23. Iacompetta M. Dissemination of technology in market and planned economies. The B.E. Journal of Macroeconomics, Contributions to Macroeconomics 2004; 4(1):Article 2.

24. Terborgh G. Dynamic Equipment Policy. Machinery and Allied Products Institute: Washington, DC, 1949.

25. Smith VL. Investment and Production: A Study in the Theory of the Capital-Using Enterprise. Harvard University Press: Cambridge, MA, 1961.

26. Malcolmson JM. Replacement and the rental value of capital equipment subject to obsolescence. Journal of Economic Theory 1975; 10:24-41.

27. van Hilten O. The optimal lifetime of capital equipment. Journal of Economic Theory 1991; 55:449-454.

28. Askenazy P, Le Van C. A model of optimal growth strategy. Journal of Economic Theory 1999; 85:24-51. 